\title{
Sensitive and non-invasive method for the in vivo analysis of membrane permeability in small animals
}

\author{
Andrea Fernandez-Carrera', Eva Vigo², Carla Regueiro-Rodríguez ${ }^{1}$, África González-Fernández', David Olivieri ${ }^{3}$ and \\ Luiz S Aroeira ${ }^{1}$
}

Tissue membranes are boundaries that isolate organs or cavities in the body. These semi-permeable membranes are responsible for passive protection that acts through the regulation of nutrient absorption, secretion and filtration of small molecules. These functions could be altered as a consequence of inflammation or trauma, which in turn could lead to changes in permeability, allowing the entrance of toxins, antigens, proteins or facilitating the spread of tumors. Membrane permeability therefore plays an important role in numerous diseases. However, current experimental techniques that are available to quantify membrane permeability in small animals have limited precision and temporal specificity. Improvements in such measurements would lead to a deeper understanding of disease pathogenesis and this may accelerate the development of specific therapies. The study reported here concerns the efficacy of a novel, non-invasive imaging analysis-based measurement method that significantly improves the quantification of tissue membrane permeability in small animals, while at the same time mitigating the adverse effects experienced by the animals under study.

Laboratory Investigation (2017) 97, 1114-1120; doi:10.1038/labinvest.2017.66; published online 24 July 2017

Membranes cover the surface of bodies and line internal body cavities. Membranes play a fundamental role in the protection of the organism and they are also involved in the secretion and absorption of substances. In general, membranes have selective permeability that can be altered by physiological and/ or pathological processes. Knowledge of the detailed parameters of these membrane permeabilities would provide important clues concerning the underlying pathophysiology of diseases and enable a deeper understanding of the biological pathways. However, present experimental methods for the measurement of membrane permeability in small laboratory animals are invasive, imprecise, and lack temporal dynamics (ie, they are often performed at a single point in time). Given the state of present techniques, the determination of the true transport rates through membranes has remained elusive and knowledge in this area is incomplete.

Peritoneal dialysis (PD) is an alternative treatment for endstage renal disease (ESRD). In this therapy, the peritoneal membrane is used as a semi-permeable membrane to filter the blood. This treatment involves the instillation of hypertonic, peritoneal dialysis solution (PDF) that generates an osmotic gradient and leads to the passage of metabolites and excess water into the peritoneal cavity. ${ }^{1}$ The non-physiological and the bio-incompatibility nature of PDF induces regenerative processes that cause morphological and functional alterations of the peritoneal membrane. The principal morphological alteration is peritoneal fibrosis, which is defined as an excessive accumulation of the extracellular matrix. ${ }^{2}$ Functional alterations manifest as Ultra-Filtration Failure (UFF). The augmented peritoneal membrane permeability, due to an increase in the number of blood vessels and the consequent larger exchange area, leads to an increase in the solute transport rate into the body. UFF can also be induced by an acute inflammatory process that increases blood vessel permeability. ${ }^{2,3}$ In patients such ultrafiltration has been determined by different methods. ${ }^{4}$

The mouse model of chronic exposure to PDF is a surrogate for peritoneal dialysis in patients who develop fibrosis and ultrafiltration failure. ${ }^{5}$ However, the measurement of UFF is more difficult to achieve using mouse model when compared to humans because mice are not uremic and because it is difficult to obtain the dialysate through the catheter, which is much finer than that used in humans. In general, the membrane permeability is determined at a single

${ }^{1}$ Immunology, Biomedical Research Center (CINBIO) (Centro Singular de Investigación de Galicia 2016-2019) and Galicia-Sur Health Research Institute (IIS-GS), University Campus, University of Vigo, Vigo, Spain; ²Endocrinology Laboratory, Biomedical Research Center (CINBIO) (Centro Singular de Investigación de Galicia 2016-2019) and Galicia-Sur Health Research Institute (IIS-GS), University Campus, University of Vigo, Vigo, Spain and ${ }^{3}$ Department of Computer Science, University of Vigo, Ourense, Spain Correspondence: Dr LS Aroeira, PhD, La Paz University Hospital Health Research Institute-IDIPAZ, Paseo de la Castellana, 261, Madrid 28046, Spain.

E-mail: Isaroeira@gmail.com

Received 30 January 2017; revised 27 April 2017; accepted 27 April 2017 
point in time through a net ultrafiltration measurement. ${ }^{6,7}$ The accuracy of this quantification method depends on the ability to drain all of the dialysate. However, fibrosis and peritoneal adhesions, which are generated in the peritoneal cavity, prevent the drainage of all peritoneal liquid and give rise to errors in the measurement. Commonly used methods also rely on statistics and a large number of animals are required to study the evolution of peritoneal deterioration and drug intervention. As a result, the methods outlined above are less than optimal.

In an effort to solve the drawbacks associated with measuring UFF and the evaluation of peritoneal membrane function, we have developed a novel in vivo fluorescence imaging method. The method is less invasive and has several intrinsic benefits. For example, the method allows the study of the temporal evolution of peritoneal membrane deterioration in the same animal. Therefore, this method opens up the possibility of being effective in monitoring disease progression from therapies in small animals and it also has potential for the measurement of other membrane permeability parameters.

\section{MATERIALS AND METHODS Animals}

Female C57BL/6 mice aged between 8 and 10 weeks were used in this study (Janvier Labs, France). Animals were housed under conventional conditions in the animal house facility at the University of Vigo. Food and water were provided ad libitum to the animals and they were kept on a $12 \mathrm{~h}$ light/dark cycle. The experimental procedure was approved by the ethics committee for animal welfare of the University of Vigo, according to National Laws RD 53/2013.

\section{Peritoneal Inflammation}

Mice were divided randomly into two groups, referred to as the control and inflamed groups. Peritoneal inflammation was induced by the injection of $200 \mu \mathrm{l}$ of incomplete adjuvant (Sigma-Aldrich, San Luis, CA, USA) into the peritoneal cavity. Three days after inflammation induction, mice were assayed for peritoneal membrane permeability. Briefly, $2 \mathrm{ml}$ of FITC-dextran (FD4, MW 4000, Sigma-Aldrich) at $5 \mathrm{mg} / \mathrm{ml}$ in phosphate buffer saline (PBS) were injected into the peritoneal cavity of mice. At different times, blood samples were collected by retro orbital plexus for the detection of FITC-Dextran in serum. Additionally, in vivo imaging was used to determine arbitrary fluorescence units of the paws and the nose. In vivo images were acquired every $30 \mathrm{~s}$ for a period of 11 or 15 min using the following conditions: f-stop: 2.5, excitation filter: $480 \mathrm{~nm}$, emission filter: $535 \mathrm{~nm}, 2 \times 2$ binning.

\section{Peritoneal Dialysis}

For peritoneal dialysis, a customized vascular access port (Access Technologies, Skokie, IL) was implanted subcutaneously, and the catheter end was introduced into the peritoneal cavity by a surgical process. Thereafter, mice were subdivided into different groups. The dialysis group was instilled daily with $2 \mathrm{ml}$ of standard PD fluid composed of $4.25 \%$ glucose (Stay Safe; Fresenius Medical care, Germany) for 30 days; the control group did not receive liquid instillation. Peritoneal permeability was determined by injection, within the peritoneal cavity, of $2 \mathrm{ml}$ FITCDextran $(5 \mathrm{mg} / \mathrm{ml})$, diluted in PDF. The concentration variation was measured in the serum of the mice at different time points. The serum concentration of FITC-dextran was determined in vitro or using in vivo imaging as described above.

\section{Serum Determination of Peritoneal Permeability}

Three days after producing peritoneal damage with incomplete adjuvant, all animals received an instillation of FITCdextran as described above. Blood was collected by the retro orbital plexus of mice at different time points. Blood $(70 \mu \mathrm{l})$ was diluted in $70 \mu \mathrm{l}$ of saline and stored at $4{ }^{\circ} \mathrm{C}$ in the dark for $4 \mathrm{~h}$ in order to produce coagulation. Blood was centrifuged $(2 \mathrm{~min}$ at $1200 \mathrm{rpm})$ and supernatants were collected and plated in a 96-well microplate (Thermo Fisher, Massachusetts, USA) to determine the serum concentration of FITC with an EnVision Multilabel Reader (Perkin-Elmer, Massachusetts, USA) (excitation of $485 \mathrm{~nm}$ and an emission wavelength of $528 \mathrm{~nm}$ ) and contrasted with the FITCdextran calibration curve. The serum from mice that did not receive FITC-dextran was used to determine the background.

\section{In vivo Determination of Serum FITC-Dextran Concentration}

All animals were anaesthetized with $100 \mu \mathrm{l}$ of a mixture of xylazine-imalgene $(16.67 \%$ xylazine $2 \%, 50 \%$ imalgene in saline) and placed in an imaging system for fluorescence detection in vivo (Carestream in-vivo $\mathrm{fx}$ pro, Bruker, MA, USA). Xilagesic $2 \%$ was obtained from Calier (Barcelona, Spain) and Imalgene $(50 \mathrm{mg} / \mathrm{ml}$ ) from Merial labs (Barcelona, Spain). Before FITC-dextran instillation and every $30 \mathrm{~s}$ thereafter, the whole body fluorescence images were taken for $11 \mathrm{~min}$ using the In-Vivo FX Pro system (f-stop: 2.5, excitation filter: $480 \mathrm{~nm}$, emission filter: $535 \mathrm{~nm}, 2 \times 2$ binning; Carestream Health, Rochester, NY). For quantitative analysis, the mean fluorescence pixel intensity from the nose and paws was measured from a region of interest (ROI). All the data analysis was performed with the Carestream Molecular Imaging Software version 5.0.2 (Carestream Health).

A fluorescence imaging and serum FITC-Dextran concentration calibration curve was obtained from a linear fitting model and this curve was used to calculate peritoneal permeability through the in vivo imaging. For this, seven mice were anesthetized and instilled with FITC-Dextran. At different time points, images were taken from different mice that were subsequently bled by cardiac puncture. The serum 
concentration of FITC-dextran was determined with an EnVision Multilabel Reader. The data for each time point were used to calculate the linear calibration curves for the paw and nose. In the subsequent experiments, image values were contrasted with the curve in order to determine the circulating concentration of FITC-dextran.

\section{Statistical Analysis}

The statistical analysis was performed using Graphpad Prism \& software for MAC. To determine the statistical significance, the slope and area under the curve of the FITC-dextran vs time of each animal were calculated using Excel and Prism, respectively. For the kinetic experiments, Kruskal-Wallis and Dunn's multiple comparison tests were performed (Figure 3). The comparison between two groups was performed using Mann-Whitney $U$-test (Figure 4). $P<0.05$ was considered significant. $\left({ }^{\star} P<0.05 ;{ }^{* *} P<0.01\right)$

\section{RESULTS}

\section{Determination of Peritoneal Permeability in Serum}

FITC-dextran has been used to determine intestinal permeability in mice models. ${ }^{8}$ In the work described here, a wellestablished fluorescent tracer was used to analyze peritoneal permeability in mice using two different experimental models, namely peritoneal dialysis and peritoneal inflammation. To study the increased permeability during peritoneal dialysis, a first group of mice was exposed daily to peritoneal dialysis fluid (PDF) for a period of 30 days through a catheter implanted in their peritoneum. One month later, the mice received FITC-dextran dissolved in PDF, blood samples were collected at different times and the fluorescence was measured. In the control group the catheter was implanted but PDF was not administered. In a second group of animals, peritoneal inflammation was induced by injection of $200 \mu \mathrm{l}$ of incomplete Freud's adjuvant (Inflamed group). Three days after the induction of peritonitis, mice received an instillation of FITC-dextran and blood samples were collected at different times. Serum was diluted and the fluorescence was determined as described in the material and methods section. The control group did not receive adjuvant injection.

Serial dilutions from FITC-dextran stock were used to obtain a linear calibration curve for the subsequent interpolation and quantification of the serum concentration of FITC. The results were plotted and straight line parameters were determined by a standard least square fitting algorithm (Figure 1a). The serum concentration of FITC-dextran in mice chronically exposed to PDF for a period of 30 days is shown in Figure $1 \mathrm{~b}$ together with the results from the control group. The results show that chronic exposure to PDF causes increased peritoneal membrane permeability when compared to the control mice group. Adjuvant-induced peritonitis also increased the peritoneal permeability (Figure 1c). These results demonstrate that FITC-dextran can be used to evaluate peritoneal membrane permeability. Nonetheless, this measurement procedure suffers from some drawbacks as an
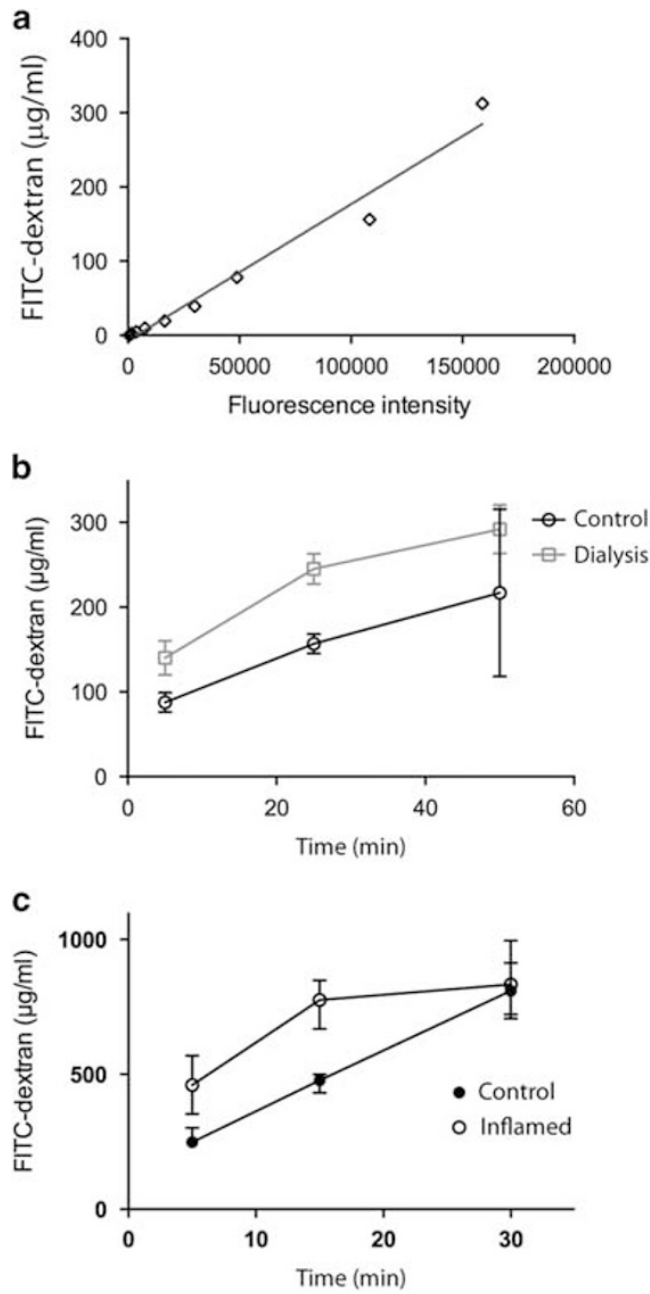

Figure 1 Quantification of plasma tracer for peritoneal permeability. A calibration curve for the FITC-dextran was prepared by serial dilution of stock (a) ( $r$ is greater than 0.97). Increased peritoneal permeability was induced in C57BL/6 mice through chronic exposure to PDF (dialysis group) (b) or by adjuvant-induced peritonitis (inflamed group) (c). After intraperitoneal administration of FITC-dextran solution, blood samples were obtained at different times. The results demonstrate that the peritoneal membrane of the dialysis group is more permeable than that in the control group (b). Compared to the control group, the peritoneal membrane of the inflamed group was shown to be more permeable to FITC-dextran (c). The results are representative of two experiments ( $n=4$ mice per group).

optimal technique to study peritoneal membrane permeability in an animal model. Firstly, the FITC-dextran diffusion rate through the peritoneal membrane cannot be measured accurately. This is because multiple measurements are required and blood volume changes and animal stress both affect the results. Therefore, the development of a new technology to measure membrane permeability is desirable.

\section{Evaluation of Peritoneal Membrane Permeability by In Vivo Imaging}

Circulating FITC-dextran could be quantified by performing in vivo imaging of vascularized and hairless body regions, such 
as the nose and paws. In order to confirm this possibility, a catheter was implanted into the peritoneum of several mice as described previously ${ }^{5}$ and 4 days later the mice were divided into two groups. The control group did not receive liquid instillation and the dialysis group received a daily instillation of PDF for a period of 30 days. On the last day of the experiment, all mice were anesthetized by a subcutaneous injection of imalgene-xylazine and an FITC-dextran solution in PDF was instilled in the peritoneum. Immediately after instillation, mice were introduced into the in vivo imaging system, and fluorescence images were acquired (every $30 \mathrm{~s}$ for a period of $11 \mathrm{~min}$ ). For quantitative analysis, fluorescence intensities from the nose and paws were measured by defining a region of interest (ROI) in the fluorescence images (Figure 2a), from which the mean pixel intensities were calculated. The variation in the pixel intensities of the ROI demonstrated that chronic exposure to peritoneal dialysis increases peritoneal membrane permeability as compared to the control group (Figure 2a). A representative example of the image pixel quantification is shown in Figure $2 \mathrm{~b}$. The data show a linear variation in the pixel intensity over time and this indicates that FITC-dextran is established in the blood circulation of mice from both groups at a constant rate during the analysis period.

In order to obtain the concentration of circulating FITCdextran for a given value of the mean ROI pixel intensity, a calibration curve was developed for different serum tracer concentrations. The procedure was as follows: normal mice were anesthetized and given an intraperitoneal injection of FITC-dextran. Images were acquired at different times from 2 to $20 \mathrm{~min}$ after injection, using the previously described imaging parameters, and the pixel fluorescence intensity from the nose and paws were recorded. Immediately afterwards, the animals were bled by cardiac puncture. The serum concentration of FITC-dextran was determined. A straight line fitting procedure was carried out and this produced a calibration curve that would provide a method for interpolating circulating FITC-dextran concentration, based upon the observed pixel intensities from the fluorescence images. An example of the FITC-dextran serum concentration $v s$ pixel intensity calibration curve obtained from the nose. Similar to the fluorescence quantification, the circulating concentration of FITC-dextran increases linearly over the time at a constant rate, as observed from the straight line fit. The slope of the line, which represents the rate of tracer diffusion through the peritoneal membrane $(\mu \mathrm{g} / \mathrm{min})$, was higher in the dialysis group than in the control group, thus demonstrating that chronic exposure to PDF increases peritoneal membrane permeability.

\section{Evolution of Peritoneal Dialysis-Induced Ultrafiltration Failure}

It is well known that continuous exposure to PDF leads to a progressive increase in peritoneal membrane permeability as a consequence of continuous angiogenesis. In order to evaluate

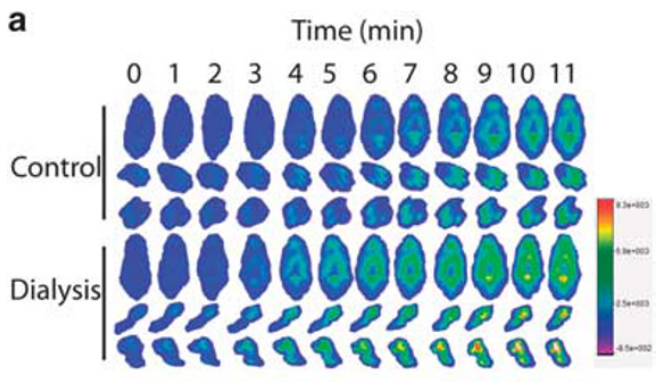

b
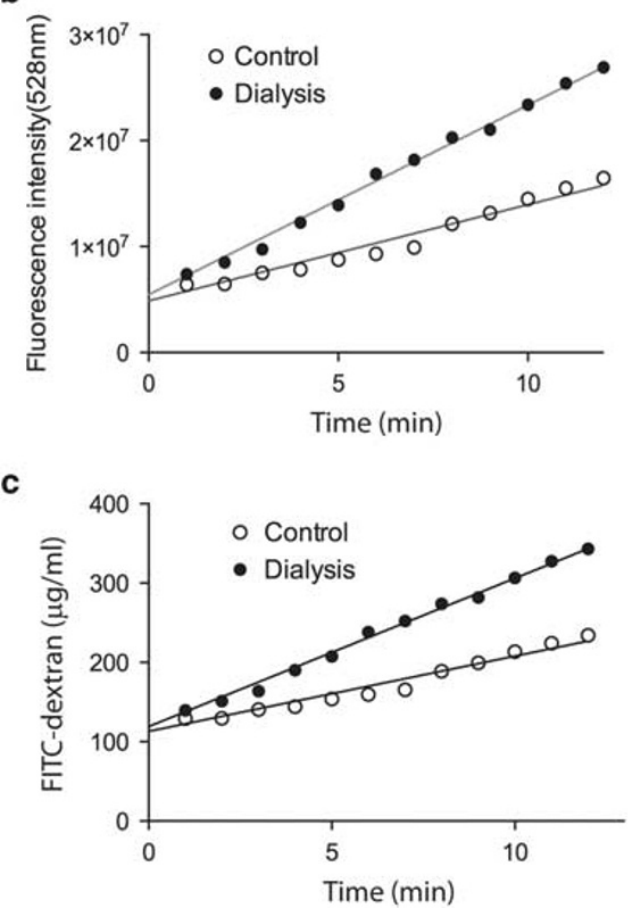

Figure 2 In vivo imaging evaluation of peritoneal permeability in mice. A selected group of mice (dialysis group) were treated with PDF during 30 days. At the end of the experiment, FITC-dextran was instilled into the mouse peritoneal cavity and body fluorescence images were acquired every $30 \mathrm{~s}$ (a). The mean pixel intensities of the ROls of the nose (above) and paws (below) were determined and demonstrated that the fluorescence intensity increased over time in both groups. However, the mean pixel intensity increased faster in the dialysis group compared to the control group (b). The in vivo calibration curve was used to determine the circulating FITC-dextran (c). The result shown is representative of two experiments ( $n=4$ mice per group).

the progression of peritoneal deterioration that leads to UFF, a catheter was inserted into each mouse. Four days after surgery, fluorescence imaging was performed prior to the instillation of a tracer (basal capture). The basal peritoneal permeability of the mice was determined by instilling FITCdextran into the peritoneal cavity and a fluorescence image was captured. Thereafter, mice received a daily instillation of PDF for 28 days. The procedure to evaluate peritoneal permeability was repeated every week. Representative fluorescence images of the nose and paws at different PDF exposure times are shown in Figure 3a. 
a

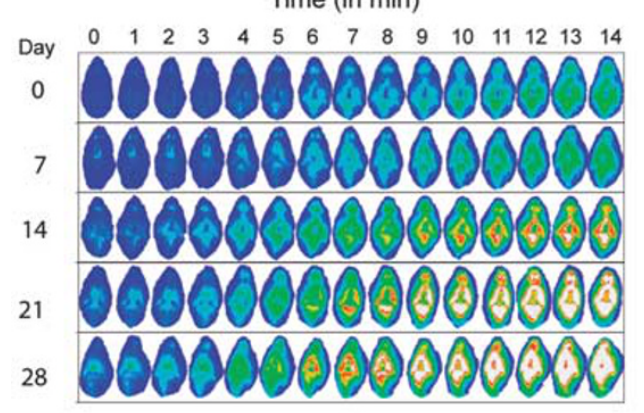

b

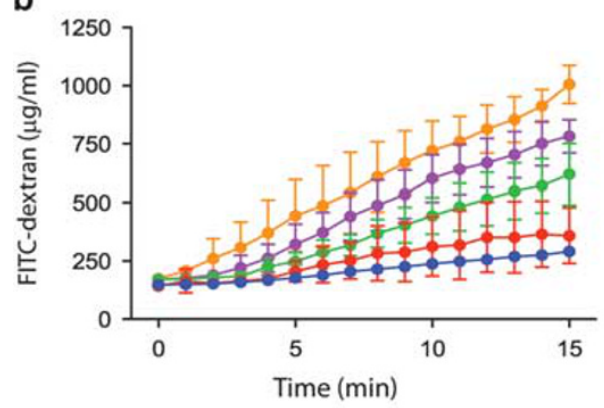

C

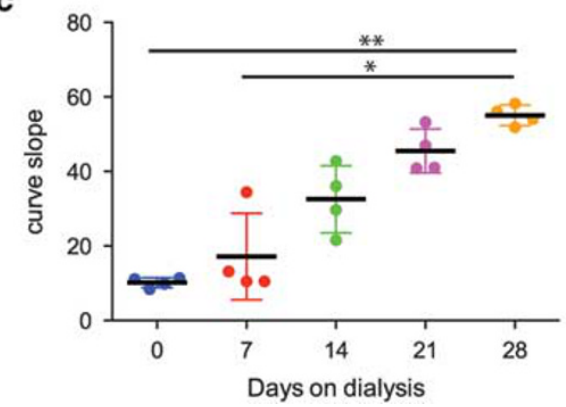

Paws

Time (in $\mathrm{min}$ )
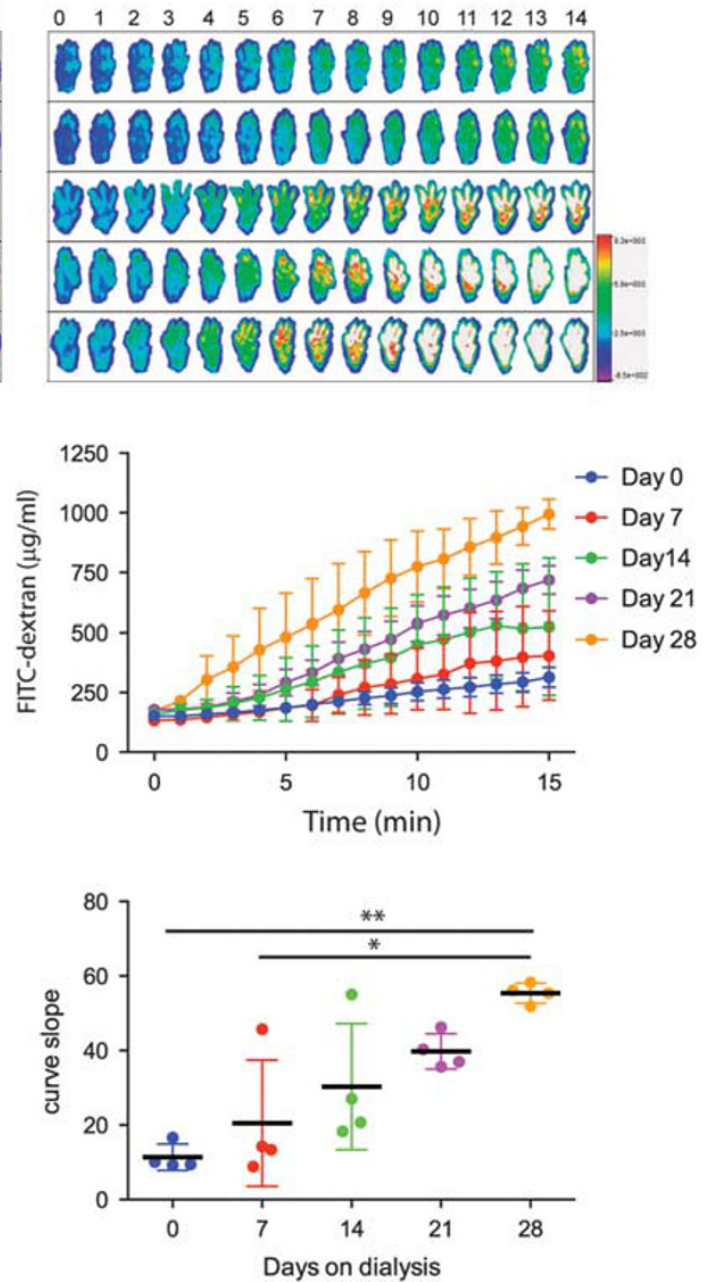

Figure 3 Kinetics of peritoneal deterioration in mice submitted to peritoneal dialysis. A catheter with a subcutaneous port was installed in all mice. After recovery from the surgery, the peritoneal membrane permeability was determined by in vivo imaging (time 0). ROI images from the nose and paws (left and right, respectively) on different days are shown (a). The circulating concentration of FITC-dextran was determined by in vivo imaging from the nose and paws (b). The calibration curve for FITC-dextran concentration over time was calculated for each mouse every week; the slope represents the rate of tracer influx into circulation $(\mu \mathrm{g} / \mathrm{min})$ for each individual calibration curve shown in $C(n=4)$. Area under the curve and slope of the curve were used for the statistical analysis, showing similar results. ${ }^{*} P<0.05$; ${ }^{*} P<0.01$.

The results demonstrate the accumulation of a small amount of FITC-dextran in the circulation of mice over time. In order to avoid the measurement of fluorescence from residual FITC-dextran instilled previously, the difference in the pixel intensity obtained prior to FITC-dextran instillation at different times of treatment and the image taken on day 0 prior to tracer instillation were subtracted from the measurement of each image obtained after FITC-dextran instillation. The result was used to calculate the circulating concentration of FITC-dextran using the in vivo calibration curve for both the nose and paws.

The results presented in Figure $3 \mathrm{~b}$ demonstrate that the circulating concentration of FITC-dextran determined from the images of nose and paws were equally efficient. In addition, these results demonstrate that the peritoneal membrane permeability progressively increased in response to chronic exposure to PDF, as demonstrated by the increasing slope of the curve with dialysis time. The individual curves for circulating tracer concentration over time for each mouse were calculated for each week. The slope of the curve, which represents the rate of tracer influx $(\mu \mathrm{g} / \mathrm{min})$, demonstrates that this method can be used to evaluate the progressive increase of peritoneal permeability as a consequence of chronic exposure to PDF (Figure 3c).

\section{Effect of Anti-Inflammatory Treatment on Ultrafiltration Failure Due to Adjuvant-Induced Peritonitis}

Adjuvant-induced peritoneal inflammation causes increased peritoneal membrane permeability. Modulation of this inflammatory process should affect the peritoneal 
a Time (min)
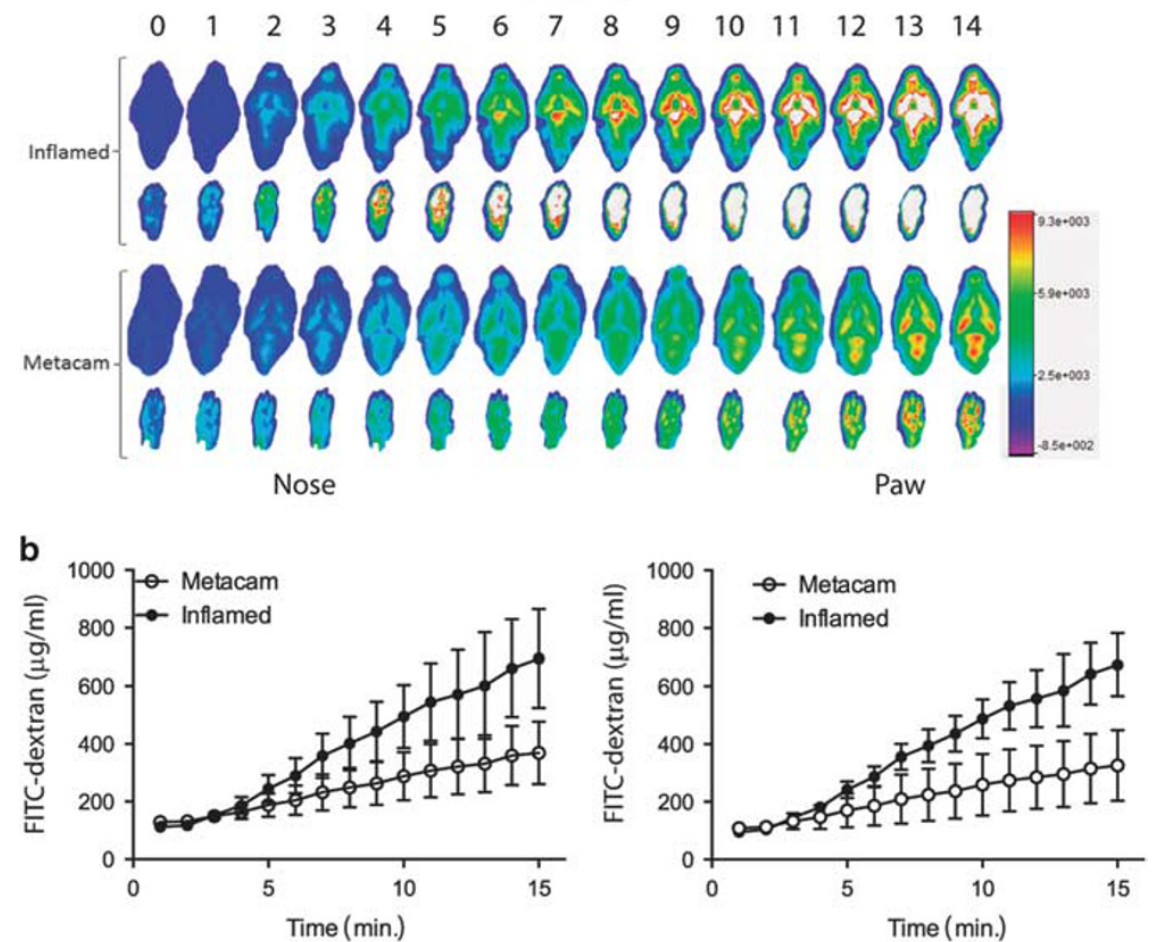

C
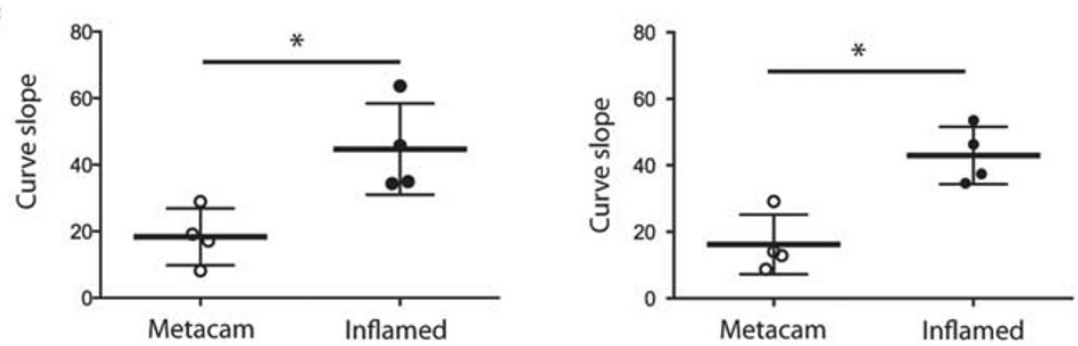

Figure 4 Metacam reduced peritoneal permeability caused by adjuvant-induced peritonitis. Incomplete Freund's adjuvant was injected into the peritoneal cavity of mice in order to induce peritonitis. Mice were randomly divided into two groups that were treated or not treated with Metacam. Representative images of the nose $(\mathrm{N})$ and paws $(\mathrm{P})$ are shown in a. The permeability of peritoneal membrane from Metacam-treated mice is lower than in the inflamed mice group (b). The calibration curve equation was determined from the results of a linear curve fitting model, where the slope for each of the mice from both groups were determined (Figure 3c) ( $n=4$ mice per group). Area under the curve and slope of the curve were used for the statistical analysis, showing similar results. ${ }^{*} P<0.05$.

permeability and this effect must be evaluated by in vivo imaging methods for the detection of circulating FITCdextran. To test the effect of Meloxicam, a non-steroidal antiinflammatory agent (cyclooxygenase inhibitor), on the ultrafiltration failures caused by adjuvant-induced peritonitis, incomplete Freund's adjuvant was introduced into the peritoneal cavity of mice. The mice were separated into two groups; one group, referred to as the 'inflamed' group, was not treated, while the other group was treated for 3 days with Meloxicam and is referred to as 'Metacam'. Four days after peritonitis induction, the mice were anesthetized and FITCdextran was injected into the peritoneal cavity. Subsequently, fluorescence in the nose and paws was analyzed. Representative ROIs of the nose and paws from both study groups are shown in Figure 4a. The circulating FITC-dextran concentration was calculated at each time point using the in vivo calibration curve obtained previously. The results demonstrate that image quantification of the circulating concentration profiles of FITC-dextran discriminate between the 'Metacam' and 'inflamed' groups (Figure 4b). Moreover, the slope of the curve confirms that the peritoneal membrane of the inflamed group was more permeable than that of the Metacam-treated group. Individual circulating concentration curves of tracer, as determined by imaging, were prepared as before and the slopes of the curves were determined (Figure 4c). The results prove that Metacam treatment reduces the rate of FITC-dextran influx through the peritoneal membrane. The findings also demonstrate that the method could also be used to evaluate therapeutic approaches. 


\section{DISCUSSION}

To date, the methods available to detect FITC-dextran in serum have provided only limited quantification of the circulating molecular concentration. In particular, some previous methods are based on measurements at a single point in time and there is no way of quantifying the flux rate through the studied membrane. If multiple measurements over time are obtained with these methods, the stress and blood volume alterations resulting from successive bleeding can influence the diffusion of FITC-dextran through the peritoneal membrane. The fluorescence in vivo imaging method described here is non-invasive and provides an accurate technique to quantify membrane permeability in small animals, whereby fluorescent molecules pass through the peritoneal membrane into the blood stream. An important advantage of this method is that it could be repeated multiple times on the same animal. The evolution of peritoneal membrane permeability monitored in the same group of mice over several weeks is shown in Figure 3.

This method is less invasive than previous methods and it can therefore elucidate individual responses to therapies and drugs by monitoring the progression of peritoneal deterioration and ultrafiltration failure over time within the same animal. Moreover, the method can also accelerate the development of a new peritoneal dialysis fluid. In addition to angiogenesis, ie, dependent ultrafiltration failure resulting from chronic exposure to PDF, the method also provides an evaluation of the effect of anti-inflammatory therapy on peritoneal membrane permeability induced by adjuvant (Figure 4). In both cases, the increase in FITC-dextran concentration in blood caused by peritoneal dialysis and adjuvant-induced peritonitis obeys a simple linear relationship, where the slope represents the entrance rate of the tracer through the membrane per minute. In addition, similar results were obtained from the measurements on images of the nose and paws, thus demonstrating that the method is consistent and reliable.

The method has some limitations that should be taken into account. Firstly, the concentration of circulating FITCdextran may be overestimated due to practical measurement capabilities during the in vivo calibration. In particular, because the blood sample is obtained $\sim 1 \mathrm{~min}$ after image acquisition, the FITC-dextran is still entering into circulation and its concentration is therefore superior to that at the time of imaging acquisition. Nonetheless, if the time difference between imaging and blood sample collection is constant, the slope of the curve will indicate the exact rate of FITC-dextran entrance into the circulation through the peritoneal membrane. Another consideration when using this method is the variation in the fluorescence intensity of FITC-dextran from different batches. More accurate measurements could be obtained by preparing calibration curves for each batch of tracer. Finally, wounds in the ROI may also affect measurements, since inflammation will cause the extravasation of the tracer and this in turn will lead to an overestimation of its circulating concentration.

Some aspects may influence the quality of image and, consequently, the sensitivity of the technique. Hair is a very autofluorescent structure that may interfere in the quality of image acquisition. Hair autofluorescence could at least be reduced by shaving or depilation with cream. ${ }^{9,10}$ However, this process may cause animal stress, mainly if experiment last long and hair should be removed more times. Depending on the type of experiment, the use of nude mice can obviate such problems. ${ }^{10}$ Tissue and body fluids also exhibit autofluorescence that could be reduced using appropriated filter as well as adjusting the contrast and brightness to improve the image quality. ${ }^{9,10}$ Alternatively, the use of longer emission wavelength probes may reduce background improving the sensibility of the technique giving a higher signal-to-background ratio.

While the method presented here is useful to evaluate peritoneal membrane permeability, it could also be used to evaluate the permeability of other membranes. For example, the permeability of the intestine could be studied by administering a tracer orally or pulmonary permeability could be evaluated by the use of an aerosol, with the circulating tracer concentration observed by in vivo image systems. This method reduces the number of animals used in the experiment and minimizes the stress to which they are exposed and it therefore complies with the three Rs principle of animal manipulation.

\section{ACKNOWLEDGMENTS}

We thank funding from Xunta de Galicia (Ministry of Culture, Education and University Management-FEDER), AFC was funded by the Spanish Ministry of Economy and Competitiveness (BES-2012-055202), and LSA and EVG were funded by BIOCAPS project (316265, FP7/REGPOT-2012-2013.1).

\section{DISCLOSURE/CONFLICT OF INTEREST}

The authors declare no conflict of interest.

1. Krediet RT, Lindholm B, Rippe B. Pathophysiology of peritoneal membrane failure. Perit Dial Int 2000;20(Suppl 4):S22-S42.

2. Krediet RT, Struijk DG. Peritoneal changes in patients on long-term peritoneal dialysis. Nat Rev Nephrol 2013;9:419-429.

3. Guo J, Xiao J, Gao H, et al. Cyclooxygenase-2 and vascular endothelial growth factor expressions are involved in ultrafiltration failure. J Surg Res 2014;188:527-36 e2.

4. Waniewski J. Peritoneal fluid transport: mechanisms, pathways, methods of assessment. Arch Med Res 2013;44:576-583.

5. Gonzalez-Mateo GT, Loureiro J, Jimenez-Hefferman JA, et al. Chronic exposure of mouse peritoneum to peritoneal dialysis fluid: structural and functional alterations of the peritoneal membrane. Perit Dial Int 2009;29:227-230.

6. Gonzalez-Mateo GT, Fernandez-Millara V, Bellon T, et al. Paricalcitol reduces peritoneal fibrosis in mice through the activation of regulatory T cells and reduction in IL-17 production. PLoS ONE 2014;9:e108477.

7. Yokoi H, Kasahara M, Mori K, et al. Pleiotrophin triggers inflammation and increased peritoneal permeability leading to peritoneal fibrosis. Kidney Int 2012;81:160-169.

8. Gupta J, Nebreda AR. Analysis of intestinal permeability in mice. Bio-protocol 2014:4:e1289.

9. Hoffman RM, Yang M. Color-coded fluorescence imaging of tumor-host interactions. Nat Protoc 2006;1:928-935.

10. Hoffman RM, Yang M. Whole-body imaging with fluorescent proteins. Nat Protoc 2006;1:1429-1438. 